\title{
Solvent Effect on Hydrogen-Bonded Acetic Acid...Arginine/Imidazole/ Phenol Dyads
}

\author{
Shihai $\mathbf{Y}^{1 *}$, Wukui Kang ${ }^{1}$ and Lixiang Sun ${ }^{2}$ \\ ${ }^{1}$ College of Chemistry and Pharmaceutical Sciences, Qingdao Agricultural University, Qingdao, China \\ ${ }^{2}$ College of Chemistry and Materials Science, Ludong University, Yantai, China
}

\begin{abstract}
The hydrogen bond plays a vital role in structural arrangement, intermediate state stabilization, and biological activity of certain enzymatic reactions. The hybrid B3LYP functional was employed with $6-311++G(d, p)$ basis set for the exploration about the solvent effect on hydrogen-bonded acetic acid...arginine, acetic acid...imidazole, acetic acid...phenol dyads, as well as the Tyr...Asp....Arg triads. The difference of two hydrogen bonds lengths in every dyad is enlarged in diethyl ether solvent as compared to that in gas phase. The increasing of solvent dielectric constant enhances the isomers' relative stability owing to the interaction between the polar conformer and the polarity solvent molecules. While the proton transfer barrier reduces. The linear relationship between dipole moment and stability can be utilized to estimate the stability modification by measuring the alteration of dipole moment of the dyad.
\end{abstract}

Keywords: Hydrogen bond; Density functional theory; Solvent effect; Potential energy surface; Proton transfer

\section{Introduction}

Hydrogen bond, which is ubiquitous in chemistry, biology, and materials, plays a fundamental role in many aspects of molecular science. According to the bond energy between a donor group (XH) and an acceptor ( $\mathrm{Y}$ ) (Both $\mathrm{X}$ and $\mathrm{Y}$ are electronegative atoms), the hydrogen bonds are classified into three types:

(i) weak or conventional with the interaction ranges from 2.4 to 12 $\mathrm{kcal} / \mathrm{mol}$;

(ii) strong or low-barrier with the coupling between 12 and $24 \mathrm{kcal} / \mathrm{mol}$;

(iii) very strong or single-well with over $24 \mathrm{kcal} / \mathrm{mol}$ interaction [1].

The hydrogen bond plays a vital role in structural arrangement, intermediate state stabilization, material function, and biological activity of certain enzymatic reactions. Especially, the successive hydrogen bonds constitute the channel for proton transfer. Generation or breakage of a hydrogen bond can produce significant changes in the kinetics or mechanism of an enzymatic reaction. The nature of the hydrogen bond in solution is of particular interest and has been probed extensively utilizing various experimental and theoretical methods [2-6].

It is reported that a strongly hydrogen bonded tyrosine residue within the active site of enzyme ketosteroid isomerase facilitates quantum proton delocalization, which dramatically stabilizes the deprotonation of the tyrosine residue, resulting in a very large isotope effect on its acidity [7]. Where, the heavy-atom distances in hydrogen-bonds are slightly longer $(\sim 2.6 \AA)$ than those typically associated with low-barrier hydrogen bonds (LBHB) [1,8,9], which have been characterized in a variety of systems [10-13]. To simulate the electron and proton transfer behavior in photosystem, the phenolimidazole-base triads have been employed with a special emphasis on solvent effect [14]. The hydrogen-bonded triads have been observed in exoglucanase $[15,16]$. The hydrogen bonds have been observed in a number of enzymes, and play a vital role in the substrate recognition, the generation of enzyme-substrate complex, the detailed reaction mechanism, as well as the formation and delivering of the product $[17,18]$.

To reveal the role of these hydrogen bonds in the enzyme, it is necessary to recognize the characteristic of this network.
The hydrogen bonded dyads, generated by acetic acid and one of phenol, imidazole, and arginine, as well as the Tyr...Asp...Arg triads are explored. Section 2 presents the computational details. The solvent effects on geometry structure, proton transfer mechanism, IR spectra, and the relative stability are compared. The results and discussion are collected in Section 3. Section 4 shows the conclusion.

\section{Computational Details}

The well-developed density functional theory (DFT) with the Becke three parameterized Lee-Yang-Parr (B3LYP) exchange correlation functionals has been proven to be successful in describing large free radicals and hydrogen bonded complexes $[14,19,20]$. All calculations were performed employing B3LYP functional with the $6-311++\mathrm{G}(\mathrm{d}$, p) basis set using a suite of Gaussian 09 programs [21]. At the same level, the vibrational frequency calculations were carried out to confirm the optimized stationary points to be local minima or transition states on the potential energy surfaces (PES). Solvent effect on geometry structure, IR spectra, and the energy profile for proton transfer was compared employing the integral equation formalism polarizable contimuum model (PCM) within the framework of the self-consistent reaction field theory (SCRF), which was well-developed and accepted in treating solvent effects of molecular properties. The solvent effects were estimated using the diethylether and water solvents with the dielectric constant of 4.2 and 78.4. The variations of geometrical structure, IR spectra, and the proton transfer energy profile upon reduction and oxidation are also detected [22-24].

\section{Results and Discussion}

The acetic acid...arginine (AA), acetic acid...imidazole (AI),

*Corresponding author: Shihai Yan, College of Chemistry and Pharmaceutical Sciences, Qingdao Agricultural University, Qingdao, 266109, China, Tel: 8653286080954; Fax: 8653286080213; E-mail: yansh@qibebt.ac.cn

Received September 23, 2016; Accepted October 05, 2016; Published October 10, 2016

Citation: Shihai Y, Kang W, Sun L (2016) Solvent Effect on Hydrogen-Bonded Acetic Acid...Arginine/Imidazole/Phenol Dyads. Med Chem (Los Angeles) 6: 604610. doi:10.4172/2161-0444.1000405

Copyright: () 2016 Shihai Y, et al. This is an open-access article distributed under the terms of the Creative Commons Attribution License, which permits unrestricted use, distribution, and reproduction in any medium, provided the original author and source are credited. 
acetic acid...phenol (AP) dyads, as well as the Tyr...Asp...Arg (TAA) triads in gas phase and diethylether solvent were explored. Here, the methyl-guanidine is employed as a model for arginine. The primary geometrical parameters optimized in gas phase, as well as those obtained in diethylether solvent (labeled with italic character) are presented in Figure 1. The IR spectra of these complexes in gas phase and solvent are collected in Figures 2 and 3 denotes the onedimensional (1D) potential energy surface of AA dyad in gas phase, as well as in the diethylether and water solvents. The corresponding $2 \mathrm{D}$ potential energy surfaces of AA and AP in gas phase and diethylether solvent are described in Figures 4 and 5, respectively. The variation of relative energy along with the dipole moment when the environment changes from gas phase into diethylether solvent is shown in Figure 6.

\section{Geometrical structure}

Three conformations (AA1 AA3) are determined according to two protons location for the acetic acid... arginine (AA) dyad. In AA1, the $\mathrm{O}^{\varepsilon 2} \ldots \mathrm{HN}^{\eta^{2}}$ hydrogen bond distance is $2.914 \AA$ (Table 1), longer than that of $\mathrm{O}^{\varepsilon 1} \mathrm{H} \ldots \mathrm{N}^{\eta^{1}}(2.649 \AA)$, which carries the characteristic of low barrier hydrogen bond (LBHB). The protons positions are opposite in AA2 as compared with those in AA1. The $\mathrm{O}^{\varepsilon 1} \ldots \mathrm{HN}^{\eta 1}$ and $\mathrm{O}^{\varepsilon 2} \mathrm{H} \ldots \mathrm{N}^{\eta 2}$ hydrogen bonds distances are 2.888 and $2.627 \AA$, respectively (Figure 1). Thus, the distance of $\mathrm{OH} \ldots \mathrm{N}$ is shorter than that of O...HN. As indicates that the larger the electronegativity of the atom covalently bonded with hydrogen is, the stronger the hydrogen bond is. Two fragments coupling through hydrogen bonds in AA1 and AA2, where two moieties remain neutral. The coupling between two fragments of AA3 varies into cation....anion electrostatic interaction. Both protons locate at nitrogen site in AA3, with short O...HN distances (2.587 and $2.636 \AA$ ) and bearing the LBHB features.
Two isomers are optimized for the acetic acid...imidazole (AI) dyad, AI1 and AI2. The bare nitrogen of the imidazole fragment acts as the hydrogen bond acceptor in both of them. No proton transferred AI conformer is found. The N...HO bond is a little longer by $\sim 0.1 \AA$ than those in AA dyads. While, the $\mathrm{CH}$...O coupling is weak, as is manifested by the longer distance of H...O (Table 2).

In the first isomer of acetic acid...phenol dyad, AP1, the hydroxyl group acts as both the proton donor and acceptor, with the $\mathrm{O}^{n} \mathrm{H}$... $\mathrm{O}^{\varepsilon 1}$ and $\mathrm{O}^{\varepsilon 2} \mathrm{H} \ldots \mathrm{O}^{\eta}$ distances of 2.729 and $2.788 \AA$, respectively. The electronegativity of $\mathrm{O}^{n}$ should be larger than $\mathrm{O}^{\varepsilon 2}$, as reflects the electron withdrawing property of benzene ring. The hydroxyl group of phenol fragment in this conformer, similar to $\mathrm{OH}$ in water, acts as the bridge during the proton relay from $\mathrm{O}^{\varepsilon 2} \mathrm{H}$ to $\mathrm{O}^{\varepsilon 1}$. Besides, the proton transferred conformer is same with AP1, and they are antipode. When $\mathrm{O}^{n}$ and the adjacent $\mathrm{CH}$ group serving as the hydrogen bond acceptor and donor, respectively, the AP2 structure is generated, with longer $\mathrm{O}^{\varepsilon 2} \mathrm{H} \ldots \mathrm{O}^{\eta} /$ $\mathrm{CH}$... $\mathrm{O}^{\varepsilon 1}$ distances $(2.832 / 3.470 \AA)$. Only one state is determined for gaseous hydrogen bonded neutral Tyr...Asp...Arg triad TAA1), where three residues are all neutral. The hydrogen bond between Tyr and Asp is longer by $0.10 \AA$ than that between Asp and Arg. The optimized CO distance in neutral triads $(1.360 \AA)$ is in good agreement with that calculated for phenol (1.370 $\AA) .{ }^{14}$ It was pointed out that the $\mathrm{CO}$ bond length was dominated by the $n$ value in $s p^{n}$ hybridization of oxygen and carbon atoms of the phenolic CO sigma bond.

The solvent effect on geometry structure is explored employing the diethylether solvent. The corresponding parameters are gathered together with those obtained in gas phase in Figure 1. Based on the initial structure utilized in gas phase optimization, all stationary<smiles>C[NH+]C(=N[IH+])[NH2+][O-]</smiles>

AA1 $\Delta \mathrm{E}: 0.0(3.3)$

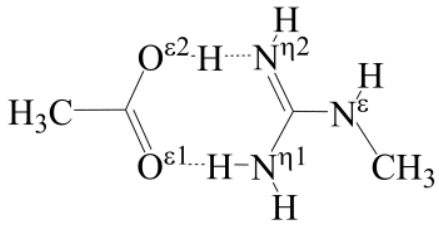

AA2 $\Delta \mathrm{E}: 1.4(4.3)$<smiles></smiles>

AA3 $\Delta \mathrm{E}: 2.1(0.0)$<smiles>CC(=O)O</smiles>

AI1 $\Delta \mathrm{E}: 0.0(0.0)$<smiles>CC(=O)O</smiles>

AI2 $\Delta$ E: $1.1(0.5)$<smiles>CC(=O)[OH+][OH+]c1ccccc1</smiles>

AP1 $\Delta \mathrm{E}: 0.0(0.0)$<smiles>CC(=[OH+])[OH+]c1ccccc1[OH+]</smiles>

AP2 $\Delta \mathrm{E}: 3.3(1.7)$

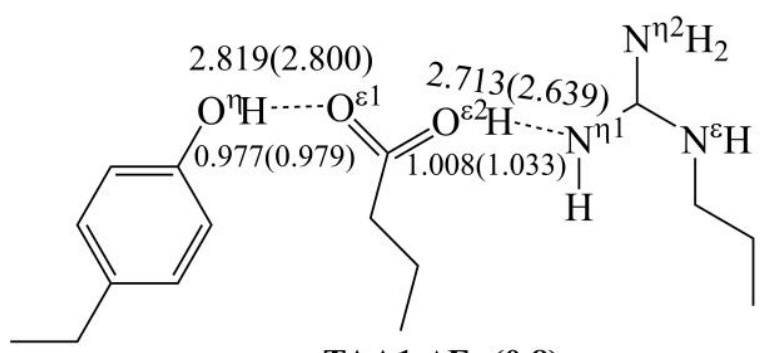

TAA1 $\Delta E:(0.8)$

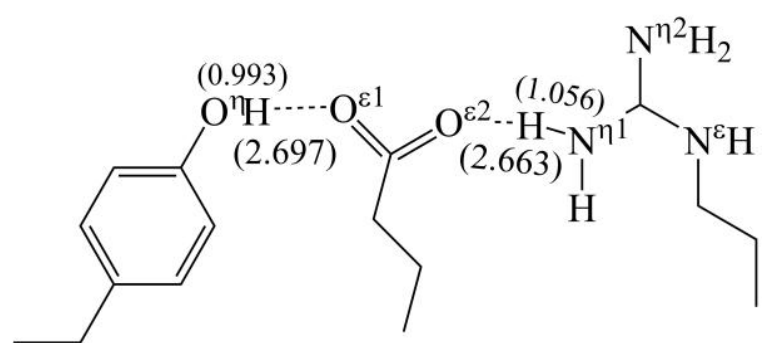

TAA2 $\Delta E:(0.0)$

Figure 1: The geometrical structures of acetic acid...arginine (AA1 AA3), acetic acid...imidazole (Al1 Al2), and acetic acid...phenol (AP1 AP2) dyads, as well as the Tyr...Asp...Arg (TAA1 TAA2) triads, accompanied with the primary parameters of triads in gas phase and diethylether solvent (in parentheses). The units for hydrogen bond length and energy are in $\AA$ and $\mathrm{kcal} / \mathrm{mol}$, respectively. 


\begin{tabular}{|c|c|c|c|c|c|c|}
\hline & \multicolumn{2}{|c|}{ AA1 } & \multicolumn{2}{|c|}{ AA2 } & \multicolumn{2}{|c|}{ AA3 } \\
\hline & Gas & Diethylether & Gas & Diethylether & Gas & Diethylether \\
\hline $\mathrm{O}^{\varepsilon 1} \mathrm{H} / \mathrm{N}^{n^{1}} \mathrm{H}$ & 1.032 & 1.058 & 1.024 & 1.022 & 1.098 & 1.056 \\
\hline $\mathrm{O}^{\varepsilon 1} \ldots \mathrm{N}^{\eta^{1}}$ & 2.649 & 2.592 & 2.888 & 2.918 & 2.587 & 2.700 \\
\hline $\mathrm{O}^{\varepsilon 2} \mathrm{H} / \mathrm{N}^{n 2} \mathrm{H}$ & 1.023 & 1.021 & 1.040 & 1.075 & 1.075 & 1.051 \\
\hline $\mathrm{O}^{\varepsilon 2} \ldots \mathrm{N}^{\eta^{2}}$ & 2.914 & 2.948 & 2.627 & 2.565 & 2.636 & 2.722 \\
\hline
\end{tabular}

Table 1: Primary geometrical parameters $(\AA)$ of acetic acid...arginine isomers optimized at B3LYP/6-311++G(d, p) basis set level.

\begin{tabular}{|c|c|c|c|c|c|c|c|c|c|}
\hline & \multicolumn{2}{|c|}{ Al1 } & \multicolumn{2}{|r|}{$\mathrm{Al} 2$} & & \multicolumn{2}{|c|}{ AP1 } & \multicolumn{2}{|c|}{ AP2 } \\
\hline & Gas & Diethylether & Gas & Diethylether & & Gas & Diethylether & Gas & Diethylether \\
\hline (C) $\mathrm{H} \ldots \mathrm{O}$ & 2.415 & 2.664 & 2.575 & 2.847 & $\mathrm{O}^{n} \mathrm{H}$ & 0.977 & 0.977 & 0.963 & 0.965 \\
\hline $\mathrm{OH}$ & 1.009 & 1.022 & 1.006 & 1.019 & $\mathrm{H} . . . \mathrm{O}^{\varepsilon 1}$ & 2.729 & 2.747 & 2.432 & 2.553 \\
\hline N...O & 2.726 & 2.676 & 2.737 & 2.686 & $\mathrm{HO}^{\varepsilon 2}$ & 0.982 & 0.982 & 0.980 & 0.982 \\
\hline & & & & & $\mathrm{O}^{n} \ldots \mathrm{O}^{\varepsilon 2}$ & 2.788 & 2.798 & 2.832 & 2.806 \\
\hline
\end{tabular}

Table 2: Primary geometrical parameters $(\AA)$ of acetic acid...imidazole and acetic acid...phenol isomers optimized at B3LYP/6-311++G(d, p) basis set level.

states are determined in diethylether solvent. Furthermore, another conformer (TAA2) with the proton transferred from Asp to Arg is appended. The $\mathrm{O}^{n} \mathrm{H} \ldots \mathrm{O}^{\varepsilon 1}$ distance in TAA2 is shorter as compared to the corresponding length in TAA1 by $\sim 0.1 \AA$. This indicates that the coupling between Tyr and Asp is strengthened along with this proton transfer. As compared with the length optimized in gas phase, the longer hydrogen bond lengthens while the shorter one shortens in diethylether solvent. In other words, the difference between two hydrogen bonds is enlarged in diethylether solvent. The gap of hydrogen bonds lengths is enhanced further with the increase of dielectric constants.

\section{IR spectra}

As a good facility, the IR spectra have been employed to determine the geometry structure because the variation of conformer can be reflected directly by the shift of IR spectra. The IR spectra of the selected dyads calculated in gas phase and diethylether solvent are presented in Figure 2. The difference of IR spectra in fingerprint region for three gaseous AA conformers is slight. Significant distinction is observed for the spectra with strong signals over $2000 \mathrm{~cm}^{-1}$. Two signals appear at 2571.6 and $3373.8 \mathrm{~cm}^{-1}$ for conformer AA1. They are assigned as the $\mathrm{O}^{\varepsilon 1} \mathrm{H} \ldots \mathrm{N}^{\eta 1}$ and $\mathrm{O}^{\varepsilon 2} \ldots \mathrm{HN}^{\eta 2}$ hydrogen bonds stretching, respectively. In AA2, the spectra with distinct signal locate at 2443.3 and 3351.3 $\mathrm{cm}^{-1}$ are assigned as the $\mathrm{O}^{\varepsilon 2} \mathrm{H} \ldots \mathrm{N}^{\eta^{2}}$ and $\mathrm{O}^{\varepsilon 1} \ldots \mathrm{HN}^{\eta 1}$ stretching modes, respectively. Two strong signals are observed in the IR spectra of gaseous conformer AA3 at 2168.9 and $2530.3 \mathrm{~cm}^{-1}$. The assignments were given as the $\mathrm{O}^{\varepsilon 1} \ldots \mathrm{HN}^{\eta 1}$ and $\mathrm{O}^{\varepsilon 2} \ldots \mathrm{H} \mathrm{N}^{\eta 2}$ hydrogen bonds stretching, respectively.

When the dyads are dipped into the diethylether solvent, the frequency of the $\mathrm{OH} . . . \mathrm{N}$ stretching mode in AA1 and AA2 is redshifted significantly, approaching to $2000 \mathrm{~cm}^{-1}$. The effect on $\mathrm{O} \ldots \mathrm{HN}$ hydrogen bond is slight. Two O...HN stretching modes are blueshifted and approach to each other in AA3 structure as compared to those in gas phase. The OH...N stretching mode in AI1 and AI2 is red shifted when the system changes from gas phase into diethylether solvent. On the contrary, the influence brought by solvent dielectric constant on the spectra of AP is slight (Figure 2).

The CO stretching mode of gaseous TAA1 is assigned at 1292.3 $\mathrm{cm}^{-1}$, which is blue-shifted slightly as compared with the CO stretching in AP1 and AP2 by 21 and $50 \mathrm{~cm}^{-1}$, respectively. The CO stretching of TAA1 red-shifts to $1281.6 \mathrm{~cm}^{-1}$ in diethylether solvent. The $\mathrm{OH}$...O and $\mathrm{OH} . . . \mathrm{N}$ stretching modes connecting three fragments are assigned in gas phase at 3570.6 and $2962.4 \mathrm{~cm}^{-1}$, respectively. The OH...N mode red-shifts distinctly in diethylether solvent to $2502.2 \mathrm{~cm}^{-1}$, while the solvent effect on $\mathrm{OH}$...O stretching is slight. These two stretching modes of TAA2 are assigned at $2775.0 \mathrm{~cm}^{-1}$ for $\mathrm{OH} . . . \mathrm{O}$ hydrogen bond and $3241.6 \mathrm{~cm}^{-1}$ for $\mathrm{O} \ldots \mathrm{HN}$ hydrogen bond, respectively.

\section{Potential energy surfaces}

The potential energy surfaces (PESs) for proton transfer of AA system in gas phase, diethylether, and water solvent were determined (Figure 3). Taken the energy of the most stable structure in every phase as zero point, the lines are described as the relationship between the relative energy and two $\mathrm{O}$...H distances.

In gas phase, the most stable structure is $\mathbf{A A 1}$, the other two conformers are higher in energy by 1.4 (AA2) and 2.1 (AA3) kcal/ mol, respectively. AA1, the predominant conformer in gas phase, corresponds to the left minima points on black and red lines at $\sim 1.05 \AA$ of $\mathrm{O} . . . \mathrm{H}$ distance $\left(\mathrm{R}_{\mathrm{O} \ldots \mathrm{H}}\right)$. AA2 and AA3 refer to the right local minima on red and black lines, respectively, approaching $1.55 \AA$ of $\mathrm{O} \ldots \mathrm{H}$ distance. The transition state, locating between AA1 and AA2/AA3, is determined with the energy higher than AA1 by $1.5 / 2.3 \mathrm{kcal} / \mathrm{mol}$. For the backward reaction, the barrier for the inversion of configuration from AA3 to AA1/AA2 is very low. In diethylether solvent, the energy of conformer AA3 is the lowest around O...H distance of $1.65 \AA$ Relatively, AA1 and AA2 are higher in energy by 3.3 and $4.3 \mathrm{kcal} /$ mol, respectively near $1.05 \AA$ of $\mathrm{R}_{\mathrm{O} \ldots \mathrm{H}}$. The energy barrier varing from high-energy conform AA1/AA2 to low-energy structure AA3 is 0.3/0.0 $\mathrm{kcal} / \mathrm{mol}$, decreasing as compared with the barrier in gas phase. The principal conformation corresponds to conformer AA3.

The increasing of solvent dielectric constant enhances the stability of polar conformer. Similar phenomenon has been observed in hydrogenbonded water chains [19]. As contributes mainly to the interaction between the polar conformer and the polarity solvent molecules. The larger the solvent dielectric constant is, the stronger the interaction is. Here, the potential energy surfaces in water solvent are also explored in order to inspect the dielectric constant effect on proton transfer. When the dielectric constant increases furthermore along with the solvent variation from diethylether to water, the minimum at left side near 1.05 $\AA$ of $R_{\mathrm{O}}$ disappears, and the double-well PES converts into the singlewell PES. No matter where the protons locate initially, both of which transfer to the Arg moiety after optimization.

To determine the proton transfer mechanism of these two protons (concerted or stepwise), the 2-dimentional potential energy surfaces in gas phase and diethylether solvent were determined (Figure 4). Three minima can be observed on top-left corner, top-right corner, and bottom-right corner in plot (a), corresponding to AA1, AA3, and AA2, 

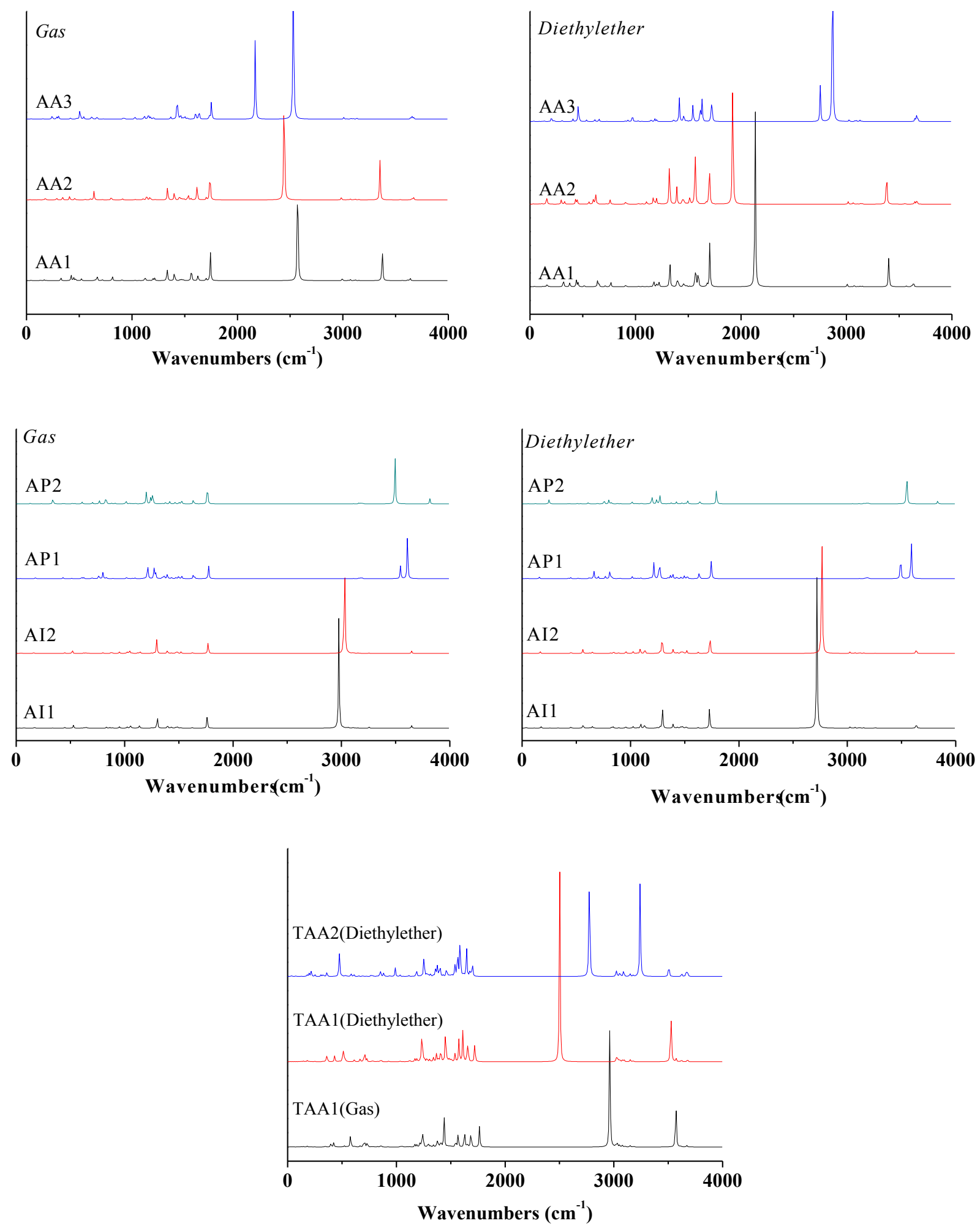

Figure 2: The IR spectra of acetic acid...arginine (AA1 AA3), acetic acid...imidazole (Al1 Al2), and acetic acid ...phenol (AP1 AP2) dyads, as well as the Tyr...Asp...Arg Triads. 
Citation: Shihai Y, Kang W, Sun L (2016) Solvent Effect on Hydrogen-Bonded Acetic Acid...Arginine/Imidazole/Phenol Dyads. Med Chem (Los Angeles) 6: 604-610. doi:10.4172/2161-0444.1000405

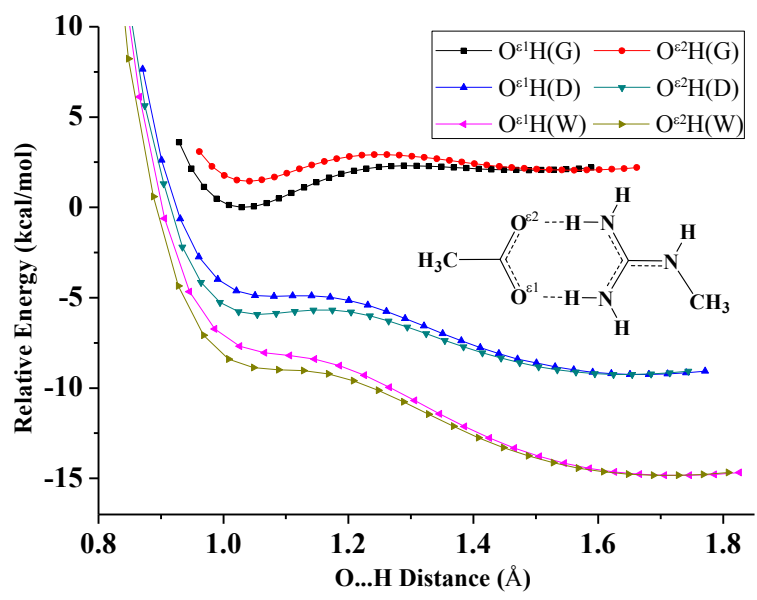

Figure 3: The 1-D potential energy surface of acetic acid...arginine (AA) dyad in gas phase $(G)$, diethylether $(D)$, and water solvent $(W)$. Arginine is simplified with a model of methyl guanidine. The energy of the most stable structure in gas phase is taken as the zero point.
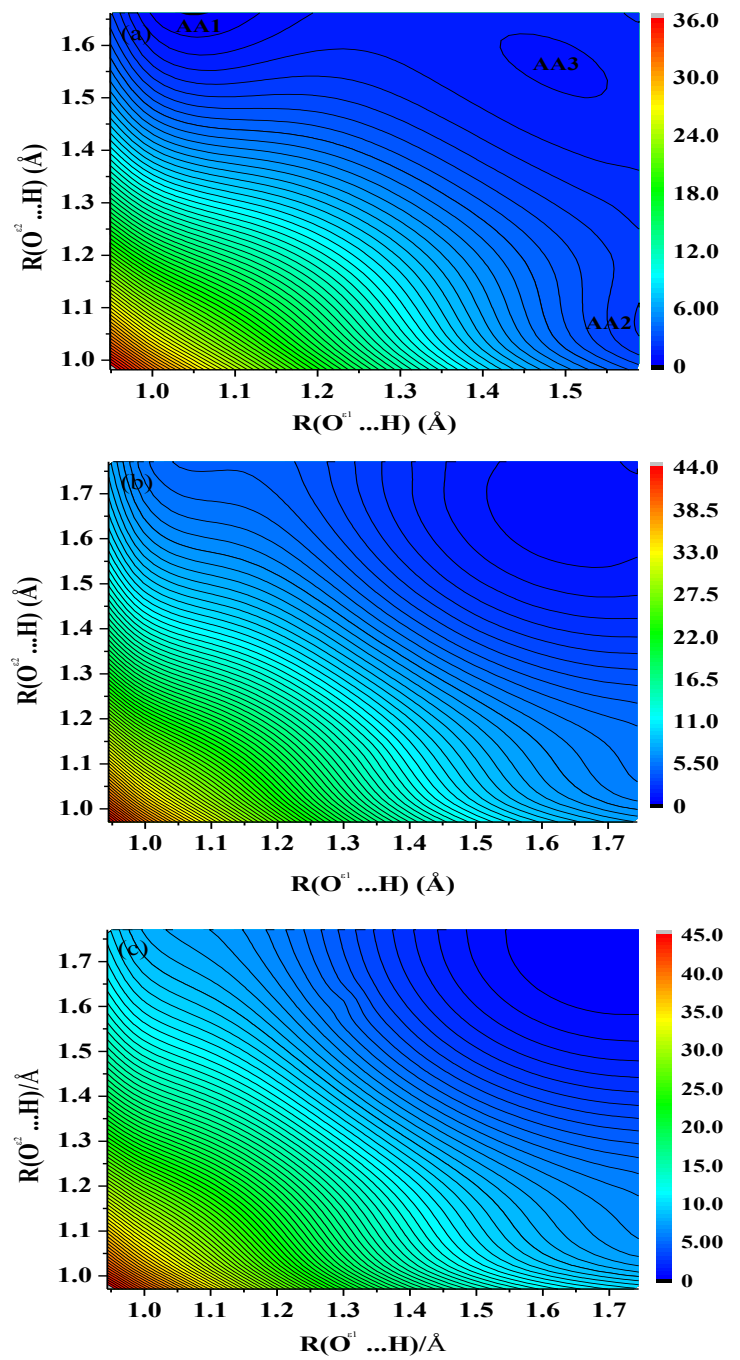

Figure 4: The 2D potential energy surfaces $(\mathrm{kcal} / \mathrm{mol})$ of acetic acid...arginine (AA) dyad in (a) gas phase; (b) diethylether, and (c) water solvent.
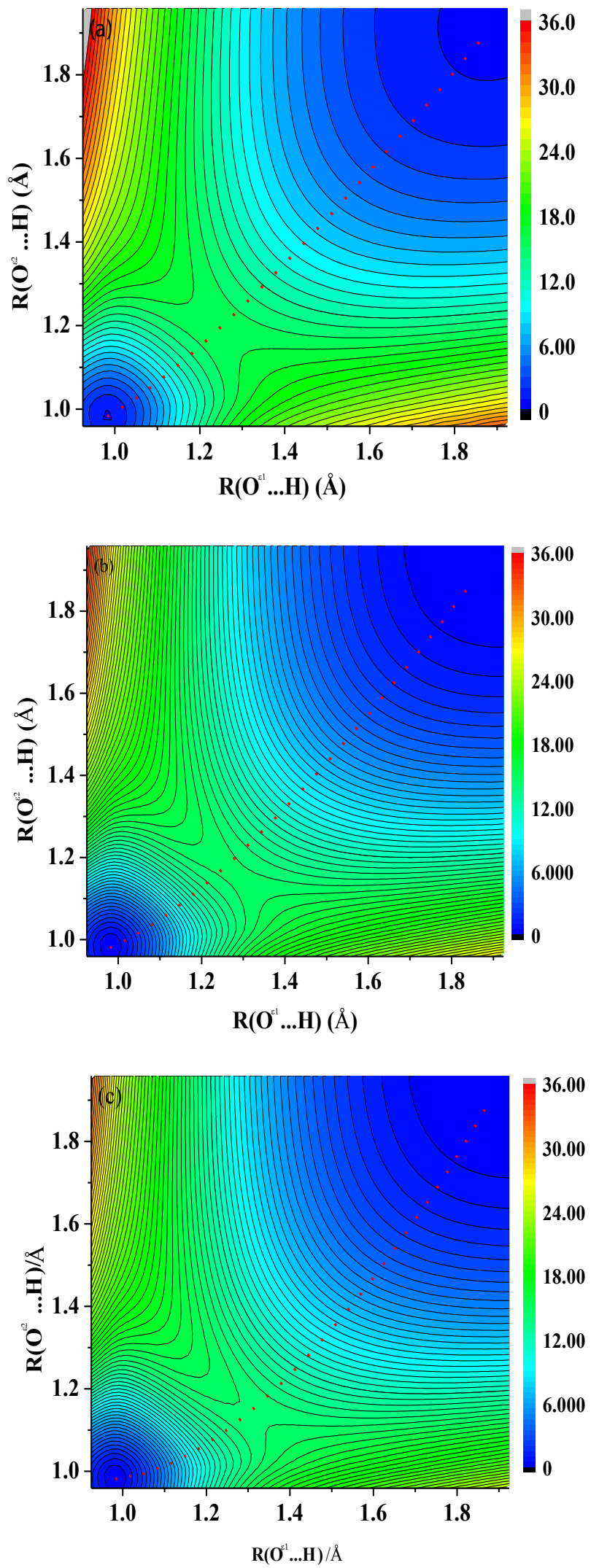

Figure 5: The 2D potential energy surfaces $(\mathrm{kcal} / \mathrm{mol})$ of acid...phenol dyad $(\mathrm{AP})$ in gas phase. 
respectively. The barriers from AA3 to AA1 and from AA3 to AA2 are relatively low as compared with that from AA2 to AA1 $(5.8 \mathrm{kcal} / \mathrm{mol})$. Therefore, these two protons transfer according to the one-by-one mechanism. The 2-dimentional PESs obtained in diethylether solvent (plot b) show obviously that AA3 corresponds to the minimum point.

The 2D PESs of acetic acid...phenol dyad in gas phase, diethylether, and water solvent shown in Figure 5 illustrate that there are two minima points, locating at bottom-left corner and top-right corner, respectively. Two protons transfer synergetically along the red dotted line, which represents the minimum energy path. Along with the increasing of solvent dielectric, the barrier of this double proton transfer decreases gradually from $\sim 22.5 \mathrm{kcal} / \mathrm{mol}$ in gas phase to 19.2 $\mathrm{kcal} / \mathrm{mol}$ in diethylether and $18.9 \mathrm{kcal} / \mathrm{mol}$ in water.

\section{Dipole Moment}

The dipole moment reflects the electrical polarity of a system. The variation of solvent dielectric constant induces the shift of dipole moment. The complexes, shown in Figure 1, were optimized in gas phase and diethylether solvent. A good linear relationship is found between the shift of the dipole moment and the variation of the relative energy (Figure 6). Such as AA1, the relative energy and the dipole moment in gas phase are $0.0 \mathrm{kcal} / \mathrm{mol}$ and 4.2 Debye, respectively. When AA1 is put into the diethylether solvent, the relative energy decreases by $5.9 \mathrm{kcal} / \mathrm{mol}$ and the dipole moment increases to 5.5 Debye. Therefore, the variation of relative energy is $5.9 \mathrm{kcal} / \mathrm{mol}$, and the alteration of the dipole moment 1.3 Debye. Thus, this relationship can be utilized to estimate the stability modification by measuring the alteration of dipole moment.

\section{Conclusion}

The calculations were carried out at B3LYP/6-311++G(d,p) level employing the polarizable contimuum model (PCM) to illustrate the solvent effect on hydrogen bonds. The difference between two hydrogen bonds distance is enlarged in diethylether solvent as compared to those in gas phase. The solvent effect on IR spectra is distinctive. The potential energy surfaces for the proton transfer of acetic acid... arginine complex in gas phase and diethylether solvent are determined. The protons transfer according to the stepwise mechanism. While, the proton transfer mechanism of acid...phenol dyad was based on the

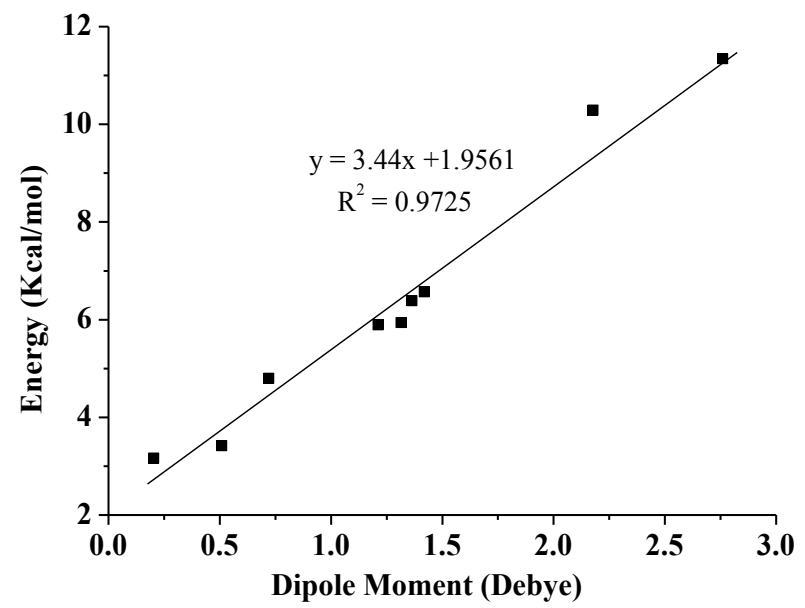

Figure 6: The variation of energy and the dipole moment when the system changes from gas phase to diethylether solvent. The energy refers to the difference of the dyad in gas phase and diethylether solvent. concerted mechanism. The increasing of solvent dielectric constant enhances the isomers' relative stability and reduces of energy barrier for proton transfer. The dipole moment can be taken as an index to judge the variation of stability.

\section{Acknowledgements}

This work is supported by National Nature Science Foundation of China (Grant No. 21203227, 21103080) and the Research Foundation for Talented Scholars of the Qing.

\section{References}

1. Frey PA, Whitt SA, Tobin JB (1994) A low-barrier hydrogen bond in the catalytic triad of serine proteases Science. Science-New York Then Washington 264: 1927-1930.

2. Perrin CL, Nielson JB (1997) Strong hydrogen bonds in chemistry and biology Annu Rev Phys Chem 48: 511-544.

3. Li P, Wang W, Sun Q, Li Z, Du A, et al. (2013) Insights into the Mechanism of the Reaction between Tetrachloro-p-Benzoquinone and Hydrogen Peroxide and their Implications in the Catalytic Role of Water Molecules in Producing the Hydroxyl Radial. Chem phy chem 14: 2737-2743.

4. Li P, Ma Z, Wang W, Song R, Zhai $Y$, et al. (2011) Theoretical studies on the electron capture properties of the $\mathrm{H}^{2} \mathrm{SO}^{-} \mathrm{HOO}^{\circ}$ complex and its implications as an alternative source of $\mathrm{HOOH}$. Physical Chemistry Chemical Physics 13 5931-5939.

5. Li $P$, Ma Z Wang $W$, Zhai $Y$, Sun $H$, et al (2011) Theoretical studies on the coupling interactions in $\mathrm{H}_{2} \mathrm{SO}_{-}-\mathrm{HOO}^{-}-(\mathrm{H} 2 \mathrm{O}) \mathrm{n}(\mathrm{n}=0-2)$ clusters: toward understanding the role of water molecules in the uptake of $\mathrm{HOO}^{\circ}$ radical by sulfuric acid aerosols. Physical Chemistry Chemical Physics 13: 941-953.

6. Li P, Shen Z, Wang W, Ma Z, Bi S, et al. (2010) The capture of ${ }^{\prime} \mathrm{H}$ and ${ }^{\circ} \mathrm{OH}$ radicals by vitamin $C$ and implications for the new source for the formation of the anion free radical. Physical Chemistry Chemical Physics 12: 5256-5267.

7. Wang L, Fried SD, Boxer SG, Markland TE (2014) Quantum delocalization of protons in the hydrogen-bond network of an enzyme active site. Proceedings of the National Academy of Sciences 111: 18454-18459.

8. Cleland WW, Kreevoy MM (1994) Low-barrier hydrogen bonds and enzymic catalysis. Science-New York Then Washington.

9. Zhao Q, Abeygunawardana C, Talalay P, Mildvan AS (1996) NMR evidence for the participation of a low-barrier hydrogen bond in the mechanism of delta 5-3-ketosteroid isomerase. Proceedings of the National Academy of Sciences 93: 8220-8224.

10. Ash EL, Sudmeier JL, De Fabo EC, Bachovchin WW (1997) A low-barrier hydrogen bond in the catalytic triad of serine proteases. Theory versus experiment Science 278: 1128-1132.

11. Kim KS, Oh KS, Lee JY (2000) Catalytic role of enzymes: Short strong H-bondinduced partial proton shuttles and charge redistributions. Proceedings of the National Academy of Sciences 97: 6373-6378.

12. Pan YP, McAllister MA (1997) The low barrier hydrogen bond in enzymatic catalysis. Journal of Biological Chemistry. J Am Chem Soc 119: 7561

13. Smallwood CJ, McAllister MA (1997) J Am Chem Soc 119: 11277.

14. Yan S, Kang S, Hayashi T, Mukamel S, Lee JY (2010) Computational studies on electron and proton transfer in phenol-imidazole-base triads. Journal of computational chemistry 31: 393-402.

15. Yan S, Li T, Yao L (2011) Mutational effects on the catalytic mechanism of cellobiohydrolase I from Trichoderma reesei. The Journal of Physical Chemistry B 115: 4982-4989.

16. Zhang Y, Yan S, Yao L (2013) A mechanistic study of Trichoderma reese Cel7B catalyzed glycosidic bond cleavage. The Journal of Physical Chemistry B 117: 8714-8722.

17. Lupas AN, Ponting CP, Russell RB (2001) On the evolution of protein folds: are similar motifs in different protein folds the result of convergence, insertion, or relics of an ancient peptide world. Journal of structural biology 134: 191-203.

18. Nigro CA, Aimaretti S (2002) J Mol Biol 320: 303

19. Kim SK, Bok JH, Bartsch RA, Lee JY, Kim JS (2005) A fluoride-selective PCT chemosensor based on formation of a static pyrene excimer. Organic letters 7: 4839-4842. 
Citation: Shihai Y, Kang W, Sun L (2016) Solvent Effect on Hydrogen-Bonded Acetic Acid...Arginine/Imidazole/Phenol Dyads. Med Chem (Los Angeles) 6: 604-610. doi:10.4172/2161-0444.1000405

20. Yan S, Zhang L, Cukier RI, Bu Y (2007) Exploration on Regulating Factors for Proton Transfer along Hydrogen-Bonded Water Chains. Chem Phys Chem 8 944-954

21. Frisch MJ, Trucks GW, Schlegel HB, Scuseria GE, Robb MA, et al. (2010) Gaussian 09. rev B 01 Gaussian Inc., Wallingford CT.

22. Miertuš S, Scrocco E, Tomasi J (1981) Electrostatic interaction of a solute with a continuum. A direct utilizaion of $A B$ initio molecular potentials for the prevision of solvent effects. Chemical Physics 55: 117-129.
23. Miertus S, Tomasi J (1982) Approximate evaluations of the electrostatic free energy and internal energy changes in solution processes. Chemical physics 65: $239-245$

24. Cossi M, Barone V, Cammi R, Tomasi J (1996) Ab initio study of solvated molecules: a new implementation of the polarizable continuum model. Chemical Physics Letters 255: 327-335. 